\title{
ANÁLISIS PROSPECTIVO DE LA GESTIÓN DEL DESTINO TURÍSTICO DE LA HABANA
}

\section{PROSPECTIVE ANALYSIS OF TOURIST DESTINATION MANAGEMENT OF HAVANA}

Yureidys García Leonard, Mgtr.

Magíster en Economía (Cuba).

Docente titular de la Universidad de la Habana, La Habana, Cuba.

yureidys@fec.uh.cu

Rafael Sorhegui Ortega, Ph.D. https://orcid.org/0000-0001-7882-5246

Doctor en Ciencias Económicas (Cuba).

Docente-Investigador del Centro de Estudios para el Desarrollo Sostenible de la Universidad ECOTEC, Samborondón, Ecuador. rsorhegui@ecotec.edu.ec

Arnaldo Vergara Romero, Mgtr. https://orcid.org/0000-0001-8503-3685

Magíster en Economía (Ecuador).

Docente-Investigador del Centro de Estudios para el Desarrollo Sostenible de la Universidad ECOTEC, Samborondón, Ecuador. avergarar@ecotec.edu.ec

Laura Nogueira Moya, Lic. Licenciada en turismo (Cuba). Investigadora de la Universidad de la Habana, La Habana, Cuba. laura@fec.uh.cu

\section{ARTÍCULO DE INVESTIGACIÓN}

Recibido: 5 de diciembre de 2020

Aceptado: 25 de febrero de 2021

\section{RESUMEN}

El turismo constituye uno de los principales pilares para el incremento del PIB tanto a escala global como nacional, razón por la cual la Organización Mundial del Turismo apuesta por un Turismo responsable e inclusivo. El virus del COVID-19, provoca una caída abrupta de los principales indicadores de la actividad turística, y aunque existe la confianza acerca de la recuperación del sector, aún queda un camino largo pues los impactos reales de la pandemia todavía están por definirse. El objetivo del artículo es demostrar a través de un caso de estudio, 
la utilidad de la prospectiva estratégica y el desarrollo de escenarios en el proceso de decisiones y planes de desarrollo turísticos (Vergara-Romero, Sorhegui-Ortega \& Garnica-Jarrin, 2020). Para ello fue de vital importancia sintetizar las principales experiencias internacionales y nacionales de la proyección estratégica y la técnica de escenarios; caracterizar el destino La Habana, usando el método prospectivo. Como resultado se obtuvieron variables claves y actores que influyen sobre dichas variables, de los cuales el MINTUR y el CITMA son los más influyentes. Con estos datos se determinaron los escenarios más probables y se seleccionó uno, con una probabilidad de ocurrencia del 59\%, para el cual se definieron 7 estrategias y 11 acciones.

Palabras claves: destino turístico, gestión de destinos, análisis prospectivo, creatividad, escenarios futuros.

\section{ABSTRACT}

Tourism as an economic activity is one of the main pillars for increasing GDP both on a global and national scale, which is why the World Tourism Organization is committed to responsible and inclusive tourism. The COVID-19 virus causes a sharp drop in the main indicators of tourism activity, and although there is confidence about the recovery of the sector, there is still a long way to go as the real impacts of the pandemic have yet to be defined. The objective of this work is to demonstrate through a case study the usefulness of strategic foresight and the development of scenarios in the decision-making process and tourism development plans. For this, it was vitally important to synthesize the main international and national experiences of strategic projection and scenario technique; characterize the Havana destination, using the prospective method. As a result, key variables and actors that influence these variables were obtained, of which MINTUR and CITMA are the most influential. With these data, the most probable scenarios were determined and one was selected, with a probability of occurrence of $59 \%$, for which 7 strategies and 11 actions were defined.

Keywords: tourist destination, destination management, prospective analysis, creativity, future scenarios.

\section{INTRODUCCIÓN}

El turismo como actividad económica es fundamental para economías en desarrollo, su importancia radica en la capacidad del sector de impulsar otras ramas de la economía, tal como apuntan Brida, London y Rojas (2014). El turismo se caracteriza por el empleo de mano de obra intensiva y un gran número de conectividad con otras industrias, así como un generador de spillover entre productores y consumidores, dicha afirmación favorece sin duda la puesta en 
práctica de una estrategia de desarrollo que mitigue los problemas estructurales de las economías menos avanzadas (Pacherres-Nolivos, Vergara-Romero \& Sorhegui-Ortega, 2020), tales como el desempleo estructural y coyuntural, la debilidad de los sistemas productivos y desde el punto de vista del turismo la incapacidad de explotar en servicio de las comunidades los atractivos naturales que poseen estos países bajo criterio sustentables e inclusivos.

Hosteltur (2019) asumiendo los resultados de un estudio de Booking.com, revela que una de las tendencias que iba a marcar la evolución del turismo en el presente año, era el desarrollo de destinos secundarios, o sea aquellos no tan conocidos, con el propósito de promoverse un turismo más sostenible y no saturar a los ya consolidados destinos mundiales. Constituyendo esto en si una oportunidad para los destinos del área geográfica de Latino América y el Caribe (Vergara-Romero, Sorhegui-Ortega \& Salvador-Guerra, 2021).

La realidad que muestra la economía mundial por los efectos de la pandemia impacta en la vida social y económica de las naciones y dentro del plano de la economía, precisamente el turismo es una de las actividades más afectadas por las restricciones a la movilidad de las personas hacia el interior y fuera de fronteras (Pacherres-Nolivos, S., Vergara-Romero, A., \& Sorhegui-Rodriguez, 2020). No obstante, la Organización Mundial del Turismo estima una recuperación del sector a medida que se logre controlar la epidemia, y la mejora de la capacidad del sector de lograr un turismo responsable (Grilli et al, 2003).

Siendo así, el desarrollo de un turismo responsable será uno de los principios que marcará la etapa por COVID-19, asumiendo como objetivos la no saturación de los destinos, un turista respetuoso con el medioambiente, mayor interacción entre el visitante y la comunidad respetando costumbres. Todo lo anterior implica oportunidades para los destinos de la región geográfica de Latinoamérica y el caribe, aunque dicha expectativa está acompañada de retos, uno de los más importantes es el desarrollo de la tecnología, Hosteltur (2019) reconoce que la tecnología seguirá jugando un papel fundamental en la decisión del turista.

Hasta el 2019, el escenario del turismo a escala planetaria se manifestaba favorable como apunta Petrizzo (2020) el 10,3 \% del PIB global pertenecía al aporte del turismo, la ya citada autora reconoce que la presencia de la COVID-19 obliga al sector incorporar estimaciones de daños directos e indirectos.

Si bien es cierto que la OMT declara que el sector puede convertirse en el impulsor de la recuperación económica en un escenario pos pandemia, Petrizzo (2020) asegura que sería una tarea compleja, en la medida que hoy día no se puede hablar del impacto real de la misma. 
En la línea del pensamiento de Petrizzo se puede aportar que, aunque se nota efectos palpables en el deterioro de indicadores macroeconómico, las economías no han podido cuantificar daños en aspectos que se escapan de los indicadores sintéticos como es el caso del PIB (Shevchenko, Shevchenko \& Zaikina, 2020).

Quedan al margen la evaluación cualitativa de aspecto como el impacto en la estructura de los mercados, los modelos de negocio y la calidad de vida. Al igual que Petrizzo (2020) sostiene la hipótesis de que el rol que jugará el turismo de cara al futuro para las economías constituirá esencial, pero necesitan ser evaluado y gestionado desde otra óptica pues en la actual situación las estrategias de los gobiernos nacionales se encuentran en contante reevaluación y ajustes en pos de aminorar los efectos inmediatos impuestos ante la actual situación.

De lo anterior se deslinda que la gestión del destino no puede ser desarrollada de forma convencional como se venía realizando hasta ahora, por ejemplo, Hosteltur (2020), plantea que varios destinos españoles parten que para planificar primero es necesario conocer los efectos, lo que permite dictar acciones para reposicionamiento. En una revisión del artículo "Los destinos se preparan para impulsar el turismo tras el coronavirus" (Hosteltur, 2020), se reconocen los problemas provocados en el sector, sin embargo, las nuevas estrategias que se proponen continúan en su mayoría siendo una apuesta a lo mismo, promover la tecnología, el desarrollo de recursos endógenos, con la inclusión claro está de los protocolos de salud.

Cabe preguntarse ante el desplome del PIB global, la pérdida de empleo, la reducción de los ingresos personales, serán suficientes estas estrategias. Ante esta situación el turismo debe de analizar aspectos como:

- $\quad$ PIB de los principales mercados emisores.

- Distribución de los ingresos en las economías nacionales.

- Análisis de los niveles de empleo.

- Estructura de los mercados turístico hacia el interior de los destinos receptores.

Pues a criterio de Petrizzo y compartido por los autores el desarrollo y la gestión del destino no dependen del buen deseo, depende factores multidimensionales que muchos de ellos rebasan los criterios locales, nacionales y regionales. Desde los inicios del año 2000 autores como Ávila y Barrado (2005) criticaban los modelos de desarrollo del turismo basado en un paradigma de turismo de masa o el fordismo, todo esto producto de un cambio en el consumidor que aposta por un turismo de calidad, diversificado y responsable, promoviendo que la planificación del 
turismo debe mostrar coherencia con la planificación local del destino (Vergara-Romero \& Moreno Silva, 2019).

En concordancia con lo expresado anteriormente se necesita poner mayor énfasis en la capacidad que tenga el turismo de imbricarse en la estrategia local de desarrollo, pues su ulterior avance como actividad económica se encuentra indisolublemente ligado a la capacidad de gestionar los destinos siendo este el espacio físico donde urge un esfuerzo mancomunado de actores endógenos (Aladag, 2020).

En esencia la idea que defiende el trabajo es que las actuales condiciones económicas, la gestión del destino turístico necesita alinearse al desarrollo local, en la medida que se logre fortalecer la pequeña y mediana empresa de esos sistemas productivos locales, de ahí que la planificación turística no puede estar deslindada de las estrategias locales (Vergara-Romero et al., 2020).

Para ello entendemos que la planeación prospectiva se vislumbra como una herramienta eficaz en aras de lograr una alineación de los objetivos del sector con los locales

Razón por la cual el trabajo persigue como objetivo aplicar la planeación prospectiva a la gestión del destino frente a los cambios que se afrontaran pos COVID- 19. Para ello se centra en el análisis de la gestión del destino y las herramientas que se emplean, tomando como caso de estudio La Habana.

\section{DESARROLLO}

Teniendo en cuenta que la unidad de análisis es el destino se parte de su definición y aunque existen varios criterios se aprecia consenso en la mayoría, para el trabajo que se presenta se asume la Ávila \& Barredo "espacio físico y social con unas determinadas características y calidades que ofrece una yuxtaposición de valores ambientales, territoriales, sociales y culturales cuyo conjunto lo convierte en algo que merece la pena experimentar, que ofrece una vivencia integral al visitante" (Avila \& Barredo, 2005, p.15).

En otras palabras, la capacidad que tiene un espacio físico y geográfico en crear experiencias para el visitante, a través de sus atractivos, para ello como bien se apunta en el concepto se deben de lograr valores territoriales, sociales y culturales que solo pueden ser creados por diversos actores en una localidad tal como apuntan Merinero y Pulido (2009) la gestión turística ya no recae solamente en las entidades turísticas, sino que el territorio asume nuevas responsabilidades. 
Manente (2009), al igual Merinero y Pulido defienden la propuesta de que la planificación de los destinos debe de ser desde la perspectiva de la planificación local, al igual que ellos, Manente reconoce que uno de los aspectos esenciales del trabajo con los actores es la relación que se establece entre ellos, para dicha autora las relaciones entre agentes, que es como lo denomina, cobra importancia bajo el concepto de cadena productivas, pues la gestión del destino tiene que ser capaz de crear valor tanto para agentes privados como públicos.

Manente (2009), establece que el análisis económico del turismo en lo relativo a la gestión del destino, debe de encargarse de potenciar el debate desde la perspectiva local, donde las estrategias deben de tener como finalidad crear valor para los agentes del destino. Tales criterios permiten afirmar que se va imponiendo como enfoque para el estudio del destino el desarrollo local (Tsai \& Chen, 2020).

Dentro de la evolución de la teoría de la gestión de los destinos y con un desarrollo conceptual exponencial se perfila como modelo de gestión los llamados destinos inteligentes. En el libro blanco de los destinos inteligentes (DTI) asume el concepto publicado por la Harvard Deusto business review en 2013 (n. ${ }^{\circ} 224$ ), y lo define como "un espacio innovador consolidado sobre la base del territorio y de una infraestructura tecnológica de vanguardia. Un territorio comprometido con los factores medioambientales, culturales y socioeconómicos de su hábitat, dotado de un sistema de inteligencia que capte la información de forma procedimental, analice y comprenda los acontecimientos en tiempo real, con el fin de facilitar la interacción del visitante con el entorno y la toma de decisiones de los gestores del destino, incrementando su eficiencia y mejorando sustancialmente la calidad de las experiencias turísticas".

Los cuatros pilares de los (DTI) son la Tecnología, innovación, accesibilidad y la sustentabilidad. Asistimos, por tanto, a un nuevo modelo de turismo integral, centrado en las personas, competitivo, creativo, sostenible y accesible. (Libro Blanco de los Destinos Inteligentes, 2016), analizando detenidamente lo que se propone como un DTI, no aporta aspectos significativos a los demás conceptos desarrollados sobre el destino, como modelo es un intento por conciliar lo tradicional con los avances recientes en la tecnología aplicada al turismo, el manejo de la Big- Data y la gestión del conocimiento.

Por otra parte, es un intento en reconocer el papel que juega la innovación en el cambio de paradigma en el modelo turístico que se viene consolidando, donde las ventajas comparativas no predominan como patrón de inserción de los destinos y una mayor apuesta a las ventajas competitivas a través de la concreción de contextos más innovadores, razón por la cual el turismo 
de sol y playa, se encuentra dentro de las modalidades de menor dinamismo en las apuestas por el desarrollo del destino (Shen, Sotiriadis \& Zhang, 2020).

Cualquier DTI debe de proponer el desarrollo de un sistema territorial (Vergara-Romero et al., 2021), sin embargo, lo que se ha desarrollado al amparo de esta expresión se ha centrado en desarrollar sistemas turísticos tecnológicos, sin platearse un sistema que integre al turista con el destino de manera que impacte en la calidad de vida y la sostenibilidad (Luqe, Zayas y Caro, 2015).

Femenia y Navarro (2018) los modelos existentes de planificación y gestión del destino no resultan adecuados y precisan de adaptarse a un nuevo escenario tecnológico. Los autores anteriores definen competencias que deben de desarrollar los organismos encargados de la Gestión del Destino y destacan: coordinación de actores internos y gestión de las crisis entre otras, imprescindible ambas en la propuesta de la investigación.

Más recientemente Ivars y Vera (2019) analizan que el cambio de los modelos de negocios plantea incógnitas para la planificación de los destinos, y para ellos consideran que el desarrollo de los DTI en la práctica ha sido promovido por iniciativas institucionales, no cuenta con una metodología consolidada que lo configure como un verdadero paradigma útil, eficiente y perdurable. Apuntan la necesidad de incorporar los problemas de resiliencia a la planificación turística (Jimber del Río et al., 2020).

Como se puede apreciar la tendencia que va marcando la gestión de los destinos en cuanto a la planificación de los mismo pasa sin lugar a duda por la necesidad de que sea un ejercicio participativo y no impulsado desde una arista institucional restrictiva a entidades turísticas. Siendo el juego de actores locales un factor decisivo (Souto-Anido et al., 2020).

En tal sentido planificar requiere trazar horizontes y deferentes periodos de plazo, la objetividad de la planificación depende de la capacidad que tengan los decisores de interpretar el futuro y como apunta Godet (2000), No existen estadísticas para el futuro, la prospectiva estratégica pone a la anticipación al servicio de la acción.

La prospectiva nos dota de las herramientas para modelar escenarios futuros "Un escenario es conjunto formado por la descripción de una situación futura y un camino de acontecimientos que permiten pasar de una situación original a otra futura. La planificación del destino a través de la prospectiva es en sí un ejercicio de prospectiva local y a criterio de Godet esta necesita aprovecharse del conocimiento local, es un ejercicio de reflexión participativa que debe de procurar una coherencia alrededor de varios proyectos para el territorio. 


\section{MATERIALES Y MÉTODOS}

Para realizar un ejercicio de planeación prospectiva aplicada a un destino turístico, se siguieron los siguientes pasos:

Análisis estructural. Se parte de delimitar las variables claves, para ello se empleó primeramente el Método Delphi. A partir del cálculo del coeficiente de experticia, el grupo de expertos quedó conformado por un total de 15 miembros los cuales evaluaron un total de 81 variables-claves propuestas, con el empleo del paquete estadístico SPSS versión 20.0, se le aplicó el coeficiente de Kendall, a las respuestas de los expertos, siendo de 0,75, lo que demuestra concordancia en sus respuestas, teniendo en cuenta el rango promedio del ordenamiento de las variables de las 81 iniciales, solo forman parte del análisis estructural 41 .

\section{RESULTADOS}

\section{Aplicación de MICMAC}

A partir de las variables presentadas en la Taba 1, se creó una matriz de impactos cruzados, y posteriormente fue evaluada por los mismos expertos que cumplimentaron la primera encuesta, definiendo el grado de influencia de cada par de variables (Khomsi, Fernandez-Aubin \& Rabier, 2020). Una vez rellenadas las encuestas, se promediaron las respuestas y esto fue lo que se exportó al programa informático MICMAC. Automáticamente este programa emitió una Matriz de Análisis Estructural de relaciones directas entre las variables, que se muestra a continuación.

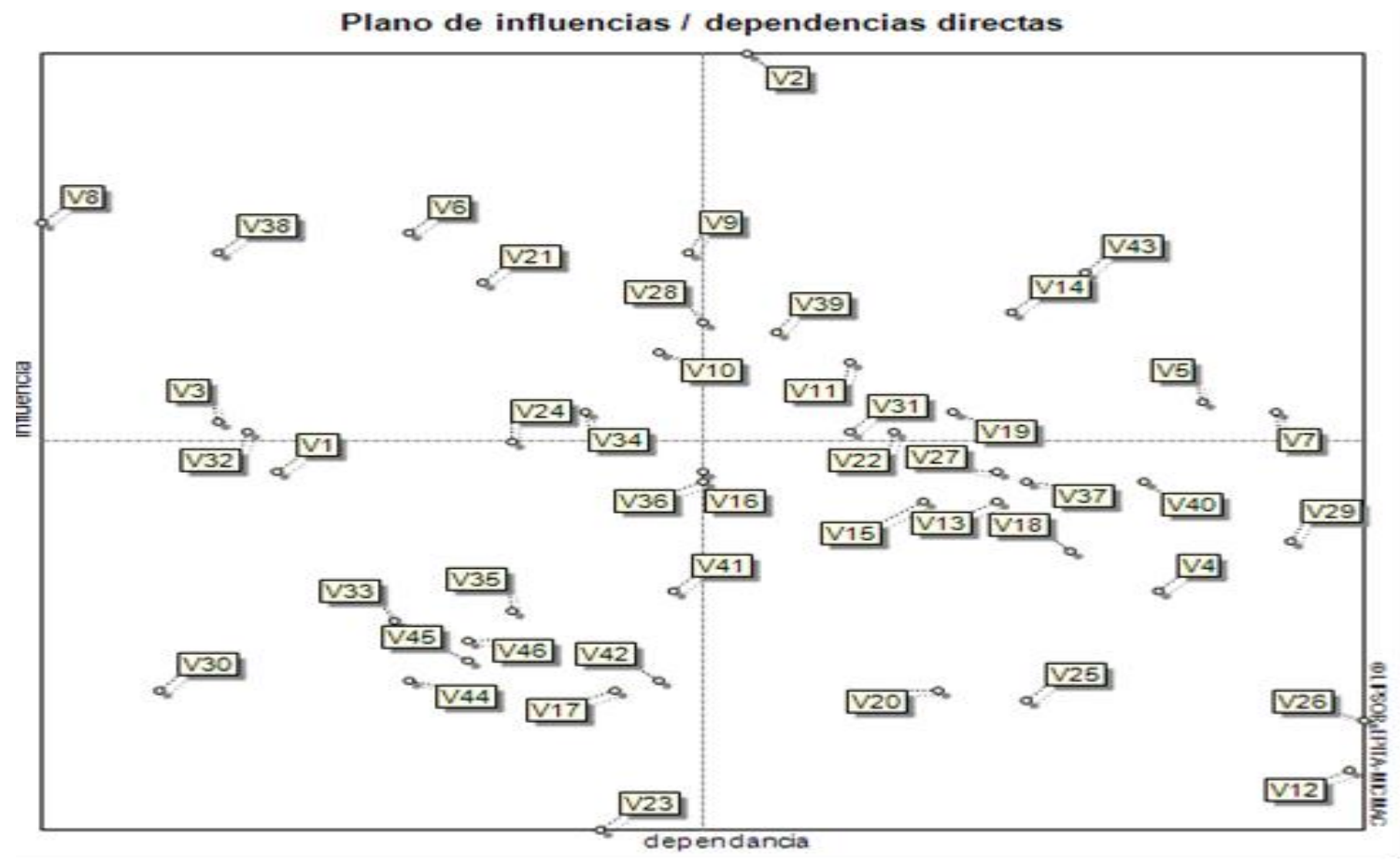

Figura 1. Resultados del método MIC-MAC. Nota. Programa MIC-MAC. Fuente: Elaboración propia. 
A continuación, se presenta las variables agrupadas teniendo en cuenta la clasificación anterior:

Tabla 1

Agrupación de variables de acuerdo a su clasificación

\begin{tabular}{|c|c|}
\hline Variables & Clasificación \\
\hline $\begin{array}{l}\text { V23 Agilidad en el pago } \\
\text { V30 Abstracción } \\
\text { V33 Fluidez }\end{array}$ & $\begin{array}{l}\text { Variables autónomas: son poco influyentes o motrices } \\
\text { y poco dependientes, se corresponden con tendencias } \\
\text { pasadas o inercias del sistema o bien están } \\
\text { desconectadas de él. No constituyen parte determinante } \\
\text { para el futuro del sistema. Se constata } \\
\text { frecuentemente un gran número de acciones de } \\
\text { comunicación alrededor de } \\
\text { estas variables que no constituyen un reto. }\end{array}$ \\
\hline $\begin{array}{l}\text { V2 Cultura de innovación } \\
\text { V6 Actualización de la tecnología } \\
\text { V8 Conocimiento de fortalezas y debilidades } \\
\text { de productos y procesos } \\
\text { V9 Imaginación } \\
\text { V21 Trabajo en equipo } \\
\text { V38 Apoyo de la dirección }\end{array}$ & $\begin{array}{l}\text { Variables determinantes: son poco dependientes y } \\
\text { muy motrices, según la evolución que sufran a lo largo } \\
\text { del periodo de estudio se convierten en frenos o motores } \\
\text { del sistema, de ahí su denominación. }\end{array}$ \\
\hline $\begin{array}{l}\text { V1 Capacidad tecnológica } \\
\text { V3 Identificación de actores para potenciar } \\
\text { tecnologías } \\
\text { V32 Reconocimiento a innovadores } \\
\text { V10 Nuevos mecanismos de cooperación y } \\
\text { alianzas estratégicas } \\
\text { V11 Oriqinalidad }\end{array}$ & $\begin{array}{l}\text { Variables de entorno: se sitúan en la parte izquierda } \\
\text { del plano, lo que demuestra su escasa dependencia del } \\
\text { sistema, hay que analizarlas como variables que reflejan } \\
\text { un "decorado" del sistema a estudio. }\end{array}$ \\
\hline $\begin{array}{l}\text { V16 Motivación } \\
\text { V22 Flexibilidad } \\
\text { V24 Infraestructura } \\
\text { V28 Coordinación entre áreas } \\
\text { V31 Creación de ambientes favorables para } \\
\text { impulsar la creatividad } \\
\text { V34 Apreciación de lo nuevo } \\
\text { V36 Comunicación } \\
\text { V39 Aceptación de cambios }\end{array}$ & $\begin{array}{l}\text { Variables reguladoras: son las situadas en la zona } \\
\text { central del plano, se convierten en "llave de paso" para } \\
\text { alcanzar el cumplimiento de las variables clave y que } \\
\text { estas vayan evolucionando tal y como conviene para la } \\
\text { consecución de los objetivos del sistema. }\end{array}$ \\
\hline
\end{tabular}


V17 Velocidad de procesamiento

V35 Participación en decisiones tecnológicas

V41 Capacidad de gestión de recursos y de estructuras de apoyo

V42 Sistema de trabajo integrador

V14 Incentivos a la innovación por los cambios tecnológicos sector

V5 Generación de productos y tecnologías propias

V7 Promoción de la creatividad

V13 Mejora de procesos

V15 Asunción de riesgos en inversión tecnológica

V18 Formas de comercializar el producto

V19 Vínculos potenciales claves

V27 Coordinación con el entorno

V29 Innovación en fórmulas de promoción y comunicación del destino

V37 Generalización de resultados innovadores

V40 Resolución de problemas no habituales
Palancas secundarias: complementarias de las

anteriores, actuar sobre ellas significa hacer evolucionar sus inmediatas anteriores: reguladoras. Se trata de variables, que, igual que las reguladoras combinan el grado de motricidad y dependencia, pero que se sitúan en un nivel inferior. Es decir, son menos motrices que las anteriores y, por lo tanto, menos importante cara a la evolución y funcionamiento del sistema, sin embargo, si las actuaciones que se acometen con ellas sirven para provocar un movimiento en las variables reguladoras, la importancia que estas variables adquieren para una adecuada evolución del sistema es evidente.

Variables-clave o variables reto del sistema: muy motrices y muy dependientes, perturban el funcionamiento normal del sistema, estas variables sobredeterminan el propio sistema. Son por naturaleza inestables y se corresponden con los retos del sistema, lo que las convierte en variables de extraordinaria importancia e integrantes. Las actuaciones que sobre ellas se vayan a tomar han de ser sopesadas con esmero, así como las que se tomen sobre aquellas que de manera indirecta se relacionan con ellas.

Variables objetivo, se ubican en la parte central son muy dependientes y medianamente motrices, de ahí su carácter de objetivos, puesto que en ellas se puede influir para que su evolución sea aquella que se desea. Su denominación viene dada porque su nivel de dependencia permite actuar directamente sobre ellas con un margen de maniobra que puede considerarse elevado, ayudando a su vez a la consecución de las variables clave. 


\section{V4 Invención}

V12 Procesos que añaden valor y productos para importar o exportar

V20 Innovación en fórmulas de relación con el cliente

V25 Usos alternativos

V26 Resultados en situaciones cambiantes
Variables resultado: se caracterizan por su baja motricidad y alta dependencia, y suelen ser junto con las variables objetivo, indicadores descriptivos de la evolución del sistema. Se trata de variables que no se pueden abordar de frente sino a través de las que depende en el sistema.

Fuente: Elaboración propia a partir de los resultados del método MIC-MAC.

Teniendo en cuenta esta clasificación se pueden decir que las variables esenciales para desarrollar el sistema y por tanto las que se tendrán en cuenta para la construcción de escenarios serán las variables claves y las variables objetivo y reguladoras, que influyen directamente sobre las claves, quedando un total de 22 variables.

\section{Identificación de actores}

Con el fin de determinar los actores que influyen en el sistema, se evaluó cada variable definida anteriormente, puntualizando así, los actores que influyen sobre las misma, los cuales se muestran a continuación.

Tabla 2

Actores y objetivos estratégicos

\begin{tabular}{|c|c|c|c|}
\hline $\begin{array}{l}\text { Nombre } \\
\text { corto del } \\
\text { actor }\end{array}$ & Actores & $\begin{array}{l}\text { Nombre } \\
\text { corto del } \\
\text { objetivo }\end{array}$ & Objetivos estratégicos \\
\hline A1 & $\begin{array}{l}\text { Ministerio de Turismo } \\
\text { (MINTUR) }\end{array}$ & O1 & $\begin{array}{l}\text { Establecer regulaciones y propuestas } \\
\text { para el desarrollo del destino. }\end{array}$ \\
\hline $\mathrm{A} 2$ & OSDEs del sector del turismo & $\mathrm{O} 2$ & $\begin{array}{l}\text { Dirigir, organizar y controlar las } \\
\text { empresas del sector. }\end{array}$ \\
\hline A3 & Entidades del sector turístico & O3 & $\begin{array}{l}\text { Comercializar el destino, haciendo } \\
\text { uso de la tecnología existente y con } \\
\text { carácter innovador. }\end{array}$ \\
\hline A4 & $\begin{array}{c}\text { Ministerio de Ciencia, } \\
\text { Tecnología y Medio Ambiente } \\
\text { de Cuba (CITMA) }\end{array}$ & $\mathrm{O} 4$ & $\begin{array}{l}\text { Dirigir, ejecutar y controlar la política } \\
\text { del Estado y del Gobierno en la } \\
\text { actividad científica y tecnológica y la } \\
\text { política ambiental. }\end{array}$ \\
\hline A5 & Gobiernos Locales & O5 & $\begin{array}{l}\text { Definir políticas de desarrollo en la } \\
\text { localidad a través de crear sinergia } \\
\text { entre actores. }\end{array}$ \\
\hline
\end{tabular}


A6 Universidad de La Habana

Escuela de Hotelería y

A7 Turismo (FORMATUR)
O6

Contribuir al desarrollo económico social, cultural y político, a través del despliegue de los procesos de formación continua de profesionales integrales, asumiendo la tecnología e innovación como aspecto relevante para la formación.

Capacitar tanto técnica como O7 idiomáticamente en las diferentes especialidades turísticas, así como la superación técnica y profesional de los cuadros y reservas de las entidades turísticas.

Fuente: Elaboración por autores.

\section{Construcción de escenarios, definición de estrategias y acciones}

Tras la determinación de las variables claves y de los actores que influyen sobre las mismas, es posible crear escenarios y definir el más probable, para posteriormente, determinar las políticas y acciones a desarrollar para alcanzarlo.

Para la construcción de escenarios se utilizó el método SMIC - Prob Expert, y el criterio de los expertos volvió a ser de suma importancia en esta etapa de definición de las probabilidades de ocurrencia de los eventos (hipótesis) en los escenarios.

Tabla 3

Resultados método SMIC-Prob-Exper

\begin{tabular}{ll}
\hline \multicolumn{1}{c}{ Variables } & \multicolumn{1}{c}{ Eventos / Hipótesis } \\
\hline V11 Originalidad & H01 Desarrollo de una cultura organizacional que \\
V16 Motivación & tenga como base la creatividad y la innovación. \\
V22 Flexibilidad & H02 Capacitación del personal en función de \\
V34 Apreciación de lo nuevo & lograr un mayor dominio tecnológico y por ende, \\
V40 Resolución de problemas no habituales & mayor capacidad de innovación. \\
V07 Promoción de la creatividad & \\
V10 Nuevos mecanismos de cooperación y alianzas & H03 Consolidación de relaciones con actores \\
estratégicas. & claves para impulsar las creatividad e innovación \\
V13 Mejora de procesos & empresarial. \\
V19 Vínculos potenciales claves & \\
V27 Coordinación con el entorno & H04 Coordinación con el entorno para adaptarse \\
V28 Coordinación entre áreas & y estar acorde con las tendencias turísticas del \\
V31 Creación de ambientes favorables & para \\
impulsar la creatividad & momento. \\
V36 Comunicación & \\
V37 Generalización de resultados innovadores & \\
V39 Aceptación de cambios &
\end{tabular}


V05 Generación de productos y tecnologías propias

V14 Incentivos a la innovación por los cambios tecnológicos sector

V15 Asunción de riesgos en inversión tecnológica

V24 Infraestructura

V18 Formas de comercializar el producto

H05 Desarrollo tecnológico avanzado, que permita estar en consonancia con las tendencias mundiales del turismo.

V29 Innovación en fórmulas de promoción y comunicación del destino

H06 Innovación en las formas de comercializar el destino, de tal modo que el producto satisfaga la mayor cantidad de segmentos de demanda

V43 Incentivos a la innovación por la demanda posibles.

Fuente: Elaboración por autores.

Tras la aplicación de las encuestas evaluando las matrices y la exportación de los datos al SMIC - Prob Expert se pudo apreciar que todas las hipótesis evaluadas, están dentro del rango de probabilidad de 70 a $80 \%$, considerándose según el criterio de expertos como eventos probables. Las Hipótesis 1, 3, 4 y 6 son las que mayores probabilidades de ocurrencia tienen dentro de ese rango.

Tabla 4

Probabilidad de ocurrencia de las Hipótesis

\begin{tabular}{|l|r|}
\hline \multicolumn{1}{|c|}{ Hipótesis } & 1- Probabilidades \\
\hline $1-\mathrm{H} 1$ & 0.858 \\
\hline $2-\mathrm{H} 2$ & 0.794 \\
\hline $3-\mathrm{H} 3$ & 0.812 \\
\hline $4-\mathrm{H} 4$ & 0.878 \\
\hline $5-\mathrm{H} 5$ & 0.775 \\
\hline $6-\mathrm{H} 6$ & 0.815 \\
\hline
\end{tabular}

Fuente: Elaboración por autores.

Finalmente, con la aplicación de este método, quedaron conformados 64 escenarios, de ellos 53 son imposibles ya que la probabilidad de ocurrencia es nula, por los que quedaron 11 posibles escenarios, como se muestra en el siguiente histograma: 


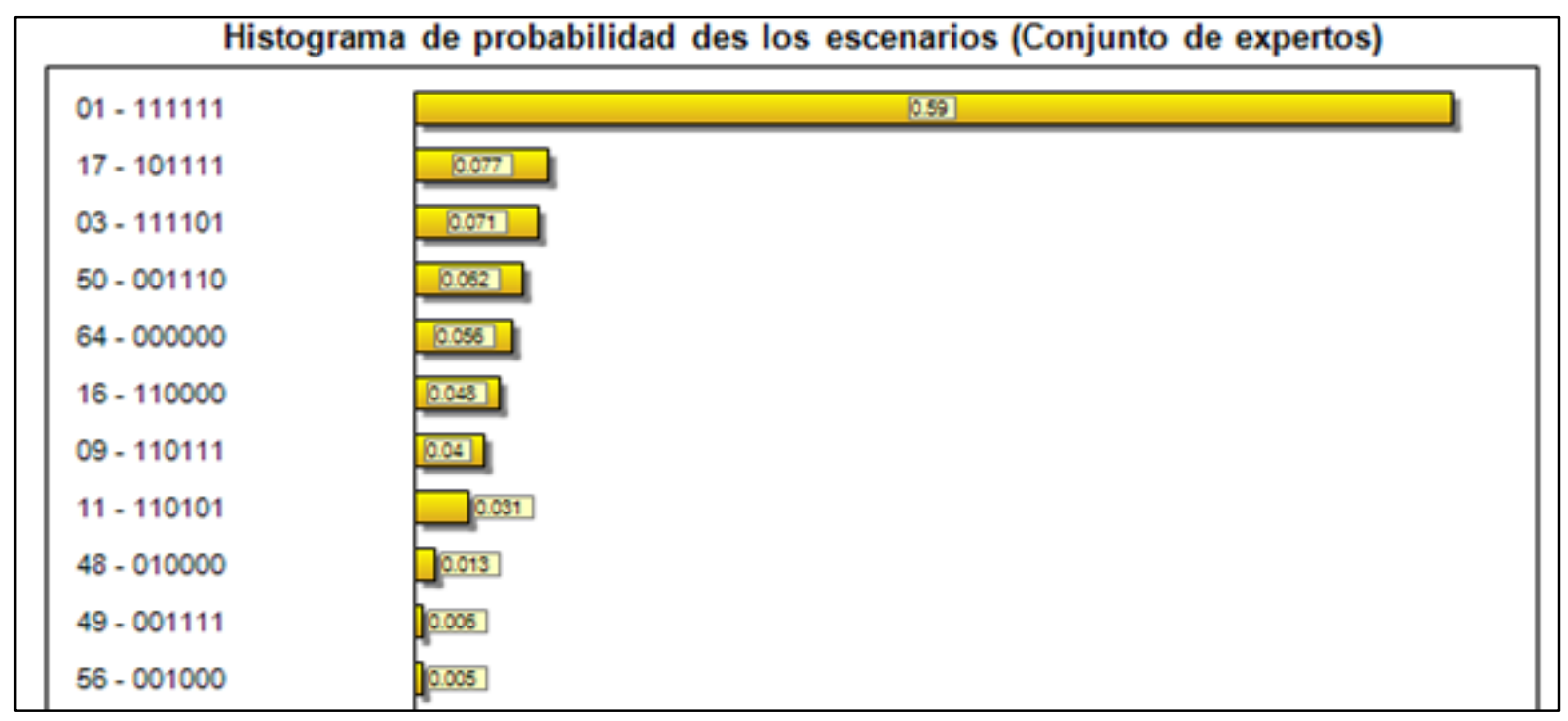

Figura 2. Histograma de probabilidad de escenarios. Nota. Program SMIC-Prob-Expert. Fuente: Elaboración propia.

De estos escenarios posibles existe uno (01 - 111111) que es el de mayor probabilidad, que es el Escenario 01 con un 59\% de ocurrencia. En este escenario influyen todas las hipótesis planteadas, y por ende es el escenario que más favorece el desarrollo de la innovación y la creatividad en el destino La Habana; por estas razones será el seleccionado para trazar las estrategias y acciones.

Siendo el escenario más probable el que pone de manifiesto la ocurrencia de las hipótesis por parte de los expertos, el destino cuenta con una herramienta eficaz que permite su planificación diseñando estrategias sobre las capacidades del destino representadas en las variables claves y utilizando la sinergia entre actores.

Como bien se observa desde el principio del trabajo la prospectiva es un método eficaz para coincidir la planeación de los destinos con lo local, pues la metodología parte de entender el fenómeno estudiado como un sistema, por lo que para el caso de estudio el destino es considerado con un sistema abierto donde se relacionan varios componentes en nuestro caso agentes económicos, sin embargo los resultados obtenidos nos demuestra que la veracidad del método depende en gran medida de la selección de experto, por lo que debe de ser uno de los pasos medulares y en el caso de ser aplicados a la localidad se deben de incluir agentes económicos, sociales, tanto privado como públicos. 


\section{CONCLUSIONES}

El turismo hasta el 2019 constituía una de las principales fuentes de ingresos tanto de países desarrollados como en vía de desarrollo, sin embargo, el escenario del 2020 con la presencia de una pandemia no se mostró alentador para la actividad, una de las más afectadas por la restricción de movimientos de las personas. No obstante, la confianza en que se convierta nuevamente en un pilar del crecimiento económico pasa sin duda por la necesidad de adaptarse a nuevos cambios en el entorno y en las conductas del viajero.

La gestión del destino turístico presenta potencialidades en ser desarrollada desde la óptica del desarrollo local, de manera que responda a principios de inclusividad, sustentabilidad en el manejo de recursos locales. En este marco la tecnología juega un rol esencial y aunque países avanzados apuestan por los llamados destinos inteligentes, el solo hecho de asimilar tecnología en el producto turístico no consiste en sí mismo una garantía de consolidar el destino en la preferencia de los viajeros.

Es por ello que la tecnología debe de convertirse en un instrumento para un fin, los llamados destinos inteligentes deben de desarrollarse en la misma línea que se fomenta el desarrollo local, que aporte al espacio físico respeto por el medio ambiente incluya a todos los actores para impactar en la calidad de vida.

La prospectiva debe de ser una herramienta asociada a la planificación en la medida que la técnica permite en si combinar actores locales, y reducir la incertidumbre del entorno con la generación de escenarios futuros.

\section{REFERENCIAS BIBLIOGRÁFICAS}

Aladag, O. F., Köseoglu, M. A., King, B., \& Mehraliyev, F. (2020). Strategy implementation research in hospitality and tourism: Current status and future potential. International Journal of Hospitality Management, $102556 . \quad$ Doi: https://doi.org/10.1016/j.ijhm.2020.102556

Arcade, J., Godet, S.M., Meunier, F. y Roubelat, F. (2004). Análisis estructural con el método MICMAC y estrategia de los actores con el método MACTOR. Laboratorio de Investigación en Prospectiva y Estrategia (LIPS). Paris.

Amozurrutia, J. (2014). Análisis prospectivo y construcción de escenarios.

Ávila Bercial, R. \& Barrado Timón, D. A. (2005). Nuevas tendencias en el desarrollo de destinos turísticos: marcos conceptuales y operativos para su planificación y gestión. Cuadernos de turismo. 
Brida, J., London, S. y Rojas, M. (2014). El turismo como fuente de crecimiento económico: impacto de las preferencias temporales de los agentes. Revista Investigación Económica, 73 (289), 59-77. Doi: https://doi.org/10.1016/S0185-1667(15)30003-5

Femenia Sierra, F. \& Navarro Ruiz, S. (2018). Identificación de fuentes de datos para la construcción de un nuevo enfoque de planificación de destinos inteligentes. Tomado de www.aceit.org.

Fundación Municipal Turismo para Cuenca. (2011). Plan Estratégico de Desarrollo Turístico del destino Cuenca y su área de influencia.

Garcés, E. (2006). Características de la Ciudad de La Habana como Destino Turístico. Tesis de maestría, Facultad de Turismo, La Habana.

Gárciga Marrero, R. J. (2001). Formulación Estratégica: un enfoque para directivos. La Habana: Felix Varela.

Godet, M. (2007). Prospectiva Estratégica: problemas y métodos. En Cuadernos de LIPSOR. Obtenido de http://prospektiker.es/prospectiva/Documentos/caja-herramientas-

Godet, M., Monti, R., Meunier, F., \& Roubelat, F. (2000). La caja de herramientas de la prospectiva estratégica. https://hal-lara.archives-ouvertes.fr/hal-02185401/document

González, N. E., Torres, I. M., \& Cala, A. S. (2018). Factors that Influence Innovation Management in Companies., XII. Obtenido de http://scielo.sld.cu/scielo.php?script=sci_serial\&pid=2306-9155\&lng=es\&nrm=iso

Grilli, G., Tyllianakis, E., Luisetti, T., Ferrini, S., \& Turner, R. K. (2003). Prospective tourist preferences for sustainable tourism development in Small Island Developing States. Tourism Management, 82, 104178. Doi: https://doi.org/10.1016/j.tourman.2020.104178

Güell, J. M. (2012). Oportunidad y conveniencia de aplicar la prospectiva al sector turismo. Estudios Turísticos (n.ำ192), pp. 7-33.

Hosteltur. (2020). Los destinos se preparan para impulsar el turismo tras el coronavirus. Tomado de www.hosteltur.com

Hosteltur (2019). Las tendencias que marcarán la demanda de viajes en el 2020. EconomíaHosteltur. www. Hosteltur.com

Ivars-Baidal, J. \& Rebollo, J. F. V. (2019). Planificación turística en España. De los paradigmas tradicionales a los nuevos enfoques: planificación turística inteligente. BAGE: Boletín de la Asociación de Geógrafos $\quad$ Españoles, 7. https://dialnet.unirioja.es/servlet/articulo?codigo=7042659 
Jimber del Río, J. A., Hernández-Rojas, R. D., Vergara-Romero, A. \& Dancausa Millán, M. (2020). Loyalty in Heritage Tourism: The Case of Córdoba and Its Four World Heritage Sites. International Journal of Environmental Research and Public Health, 17(23), 8950. https://doi.org/10.3390/ijerph17238950

Khomsi, M. R., Fernandez-Aubin, L., \& Rabier, L. (2020). A prospective analysis of overtourism in Montreal. Journal of Travel \& Tourism Marketing, 1-14. Doi: https://doi.org/10.1080/10548408.2020.1791782

Luque Gil, A. M., Zayas Fernández, B. \& Caro Herrero, J. L. (2015). Los Destinos Turísticos Inteligentes en el marco de la Inteligencia Territorial: conflictos y oportunidades.

Manente, M. (2009). Gestión de destino y antecedentes económicos: definición y supervisión destinos turísticos locales. Tomado de www.webuntwo.s3.amazonaws.com

Márquez, R. H. (s/a). La planificación estratégica territorial en la gestión del desarrollo local. Centro de Investigaciones Psicológicas y Sociológicas Ministerio de Ciencia, Tecnología y Medio Ambiente.

O.H, I. (26 de 01 de 2015). La importancia de la innovación en el sector turístico. Obtenido de https://www.hosteltur.com/comunidad/003728_la-importancia-de-la-innovacion-en-elsector-turistico.html\#ivanzar

Pacherres-Nolivos, S., Vergara-Romero, A., \& Sorhegui-Ortega, R. (2020). Responsabilidad social corporativa y el turismo sostenible. Revista Científica ECOCIENCIA, 7(2), 1-20. https://doi.org/10.21855/ecociencia.72.340

Pacherres-Nolivos, S., Vergara-Romero, A. \& Sorhegui-Rodríguez, R. (2020). Repensando el concepto de arte a través del turismo: el turismo de arte callejero en Guayaquil. RES NON VERBA REVISTA CIENTÍFICA, 10(2), 136-153.

Petrizzo, M. A. (2020). Impacto de la COVID-19 en el sector turismo. Apuntes para su esbozo. Tomado de www.researchgate.net

Rodríguez, R. M., \& Fernández, J. I. P. (2009). Desarrollo turístico y dinámica relacional. Metodología de análisis para la gestión activa de destinos turísticos. Cuadernos de turismo, (23), 173-194.

Shen, S., Sotiriadis, M., \& Zhang, Y. (2020). The Influence of Smart Technologies on Customer Journey in Tourist Attractions Within the Smart Tourism Management Framework. Sustainability, 12(10), 4157. Doi: https://doi.org/10.3390/su12104157 
Shevchenko, N. N., Shevchenko, V. I. \& Zaikina, E. N. (2020). Modern Approaches to the Formation of Professional Competencies of Prospective Experts of the Hospitality Industry. Revista TURISMO: Estudos e Práticas, (4).

Souto-Anido, L., Vergara-Romero, A., Marrero-Anciza, Y. \& Márquez-Sánchez, F. (2020). Incidencia de la Gestión de los Recursos Humanos en los resultados Organizacionales: ¿mito o realidad? GECONTEC: Revista Internacional de Gestión del Conocimiento y la Tecnología, 8(1), 1-23.

Tsai, T. H., \& Chen, C. M. (2020). An ex-ante evaluation of marketing policies to improve itinerary service. Current Issues in Tourism, 1-24. Doi: https://doi.org/10.1080/13683500.2020.1806792

Vergara-Romero, A., \& Moreno Silva, A. (2019). Soberanía alimentaria en Ecuador: fundamentos teóricos y metodológicos para un modelo de medición. Revista Científica ECOCIENCIA, 6, 1-18. https://doi.org/10.21855/ecociencia.60.256

Vergara-Romero, A., Olalla Hernández, A., Yturralde, J.M., \& Sorhegui-Ortega, R. (2020). Responsabilidad social corporativa y su impacto en el rendimiento económico de las principales Empresas en Ecuador. Revista ESPACIOS, 41(10).

Vergara-Romero, A., Sorhegui-Ortega, R. \& Garnica-Jarrin, L. (2020). Factores de desarrollo local desde el enfoque de la gestión organizativa. Revista de la Universidad del Zulia, 11(31), 86-96. http://dx.doi.org/10.46925//rdluz.31.07

Vergara-Romero, A., Sorhegui-Ortega, R. \& Salvador-Guerra, C. (2021). La Soberanía Alimentaria en el Desarrollo Local. Revista de la Universidad del Zulia, 12(32), 54-69. http://dx.doi.org/10.46925/rdluz.32.05

Vergara-Romero, A., Márquez Sánchez, F., Sorhegui-Ortega, R. \& Olalla-Hernández, A. (2021). Capital humano: Actor central para la sostenibilidad organizacional. Revista Venezolana de Gerencia (RVG), 26(93), 297-307. https://doi.org/10.37960/rvg.v26i93.34984 
Tabla 5

Variables evaluadas por los expertos como importantes para la gestión del destino La Habana.

\begin{tabular}{|c|c|c|}
\hline $\begin{array}{l}\text { Nombre de } \\
\text { la variable }\end{array}$ & Nombre largo de la variable & Descripción \\
\hline V1 & Capacidad tecnológica & $\begin{array}{l}\text { Posesión de tecnología necesaria para el buen } \\
\text { funcionamiento de la organización. }\end{array}$ \\
\hline V2 & Cultura de innovación & $\begin{array}{l}\text { Incorporación del proceso innovador en la mente de } \\
\text { las personas y actuación en correspondencia. }\end{array}$ \\
\hline V3 & $\begin{array}{l}\text { Identificación de actores para } \\
\text { potenciar tecnologías }\end{array}$ & $\begin{array}{l}\text { Personas u organismos que faciliten el acceso a la } \\
\text { tecnología }\end{array}$ \\
\hline V4 & Invención & $\begin{array}{l}\text { Capacidad de generar ideas novedosas sin } \\
\text { precedentes }\end{array}$ \\
\hline V5 & $\begin{array}{l}\text { Generación de productos y } \\
\text { tecnologías propias }\end{array}$ & $\begin{array}{l}\text { Disponibilidad de productos auténticos, diferentes a la } \\
\text { competencia }\end{array}$ \\
\hline V6 & Actualización de la tecnología & $\begin{array}{l}\text { Acceso a tecnología de punta que facilite el desarrollo } \\
\text { de la creatividad. }\end{array}$ \\
\hline V7 & Promoción de la creatividad & Estimular e incentivar el desarrollo de la creatividad \\
\hline V8 & $\begin{array}{l}\text { Conocimiento de fortalezas y } \\
\text { debilidades de productos y } \\
\text { procesos }\end{array}$ & $\begin{array}{l}\text { Dominio vasto sobre la organización a la hora de la } \\
\text { toma de decisiones }\end{array}$ \\
\hline V9 & Imaginación & $\begin{array}{l}\text { Permite manipular la información generada } \\
\text { intrínsecamente }\end{array}$ \\
\hline V10 & $\begin{array}{l}\text { Nuevos mecanismos de } \\
\text { cooperación y alianzas } \\
\text { estratégicas }\end{array}$ & Garantía de estar en consonancia con el entorno \\
\hline V 11 & Originalidad & Diferenciación y autenticad \\
\hline V12 & $\begin{array}{l}\text { Procesos que añaden valor y } \\
\text { productos para importar o } \\
\text { exportar }\end{array}$ & Creación de productos con valor añadido \\
\hline V13 & Mejora de procesos & $\begin{array}{l}\text { Potenciación de los puntos fuertes y reducción de } \\
\text { deficiencias }\end{array}$ \\
\hline V14 & $\begin{array}{l}\text { Incentivos a la innovación por } \\
\text { los cambios tecnológicos en el } \\
\text { sector }\end{array}$ & $\begin{array}{l}\text { Estímulo a la actividad innovadora asociada a la } \\
\text { tecnología en el sector }\end{array}$ \\
\hline
\end{tabular}




\begin{tabular}{|c|c|c|}
\hline V15 & $\begin{array}{l}\text { Asunción de riesgos en } \\
\text { inversión tecnológica }\end{array}$ & $\begin{array}{l}\text { Toma de decisión de invertir en tecnología en función } \\
\text { de los cambios en el entorno }\end{array}$ \\
\hline V16 & Motivación & Estímulo para desarrollar algo con interés y diligencia. \\
\hline V17 & Velocidad de procesamiento & $\begin{array}{l}\text { Capacidad de análisis y generación de soluciones de } \\
\text { manera rápida }\end{array}$ \\
\hline V18 & $\begin{array}{l}\text { Formas de comercializar el } \\
\text { producto }\end{array}$ & $\begin{array}{l}\text { Utilización de vías novedosas para comercializar los } \\
\text { productos }\end{array}$ \\
\hline V19 & Vínculos potenciales claves & $\begin{array}{l}\text { Relaciones con organizaciones que pueden influir } \\
\text { decisivamente en el quehacer de la empresa }\end{array}$ \\
\hline V20 & $\begin{array}{l}\text { Innovación en fórmulas de } \\
\text { relación con el cliente }\end{array}$ & Originalidad en la relación cliente - empresa \\
\hline V21 & Trabajo en equipo & Coordinación de ideas y esfuerzos conjuntos \\
\hline V22 & Flexibilidad & $\begin{array}{l}\text { Es la capacidad de cambiar con facilidad de un tipo de } \\
\text { pensamiento a otro }\end{array}$ \\
\hline V23 & Agilidad en el pago & $\begin{array}{l}\text { Mecanismos para agilizar el pago y garantizar } \\
\text { cumplimiento de contratos. }\end{array}$ \\
\hline V24 & Infraestructura & $\begin{array}{l}\text { Conjunto de elementos o servicios que se consideran } \\
\text { necesarios para la creación y funcionamiento de una } \\
\text { organización }\end{array}$ \\
\hline V25 & Usos alternativos & $\begin{array}{l}\text { Un pensamiento creativo desarrollado es capaz de } \\
\text { encontrar a cualquier objeto un uso que no es el } \\
\text { habitual }\end{array}$ \\
\hline V26 & $\begin{array}{l}\text { Resultados en situaciones } \\
\text { cambiantes }\end{array}$ & $\begin{array}{l}\text { Búsqueda de alternativas para salir adelante en } \\
\text { situaciones cambiantes del entorno }\end{array}$ \\
\hline V27 & Coordinación con el entorno & $\begin{array}{l}\text { Concertar medios, esfuerzos para emprender } \\
\text { acciones comunes. }\end{array}$ \\
\hline V28 & Coordinación entre áreas & $\begin{array}{l}\text { Relación coherente entre las áreas y fluidez de la } \\
\text { información. }\end{array}$ \\
\hline V29 & $\begin{array}{l}\text { Innovación en fórmulas de } \\
\text { promoción y comunicación del } \\
\text { destino }\end{array}$ & $\begin{array}{l}\text { Exploración de nuevas vías de llegar a los segmentos } \\
\text { de demanda metas. }\end{array}$ \\
\hline V30 & Abstracción & $\begin{array}{l}\text { Pasar tanto de lo general a lo particular como de lo } \\
\text { particular a lo general }\end{array}$ \\
\hline
\end{tabular}




\begin{tabular}{|c|c|c|}
\hline V31 & $\begin{array}{l}\text { Creación de ambientes } \\
\text { favorables para impulsar la } \\
\text { creatividad }\end{array}$ & $\begin{array}{l}\text { Espacios armónicos donde la imaginación y las ideas } \\
\text { fluyan. }\end{array}$ \\
\hline V32 & Reconocimiento a innovadores & $\begin{array}{l}\text { Identificación de personas u organizaciones con } \\
\text { grandes capacidades innovadoras }\end{array}$ \\
\hline V33 & Fluidez & $\begin{array}{l}\text { Es la cantidad de ideas que eres capaz de generar en } \\
\text { un tiempo determinado }\end{array}$ \\
\hline V34 & Apreciación de lo nuevo & $\begin{array}{l}\text { Saber diferenciar la transformación que se ha } \\
\text { generado en el proceso creador }\end{array}$ \\
\hline V35 & $\begin{array}{l}\text { Participación en decisiones } \\
\text { tecnológicas }\end{array}$ & Modo de incentivar el desarrollo de la tecnología \\
\hline V36 & Comunicación & Comunicación entre las áreas y con el entorno \\
\hline V37 & $\begin{array}{l}\text { Generalización de resultados } \\
\text { innovadores }\end{array}$ & Aplicación de innovaciones en todo el sector \\
\hline V38 & Apoyo de la dirección & $\begin{array}{l}\text { Voto de confianza y apoyo a las decisiones tomadas } \\
\text { por directivos de menor categoría, así como } \\
\text { trabajadores con ideas innovadoras }\end{array}$ \\
\hline V39 & Aceptación de cambios & Asumir los cambios y adaptarse a ellos \\
\hline V40 & $\begin{array}{l}\text { Resolución de problemas no } \\
\text { habituales }\end{array}$ & $\begin{array}{l}\text { Enfrentamiento a problemas poco comunes y } \\
\text { búsqueda de soluciones eficaces }\end{array}$ \\
\hline V41 & $\begin{array}{l}\text { Capacidad de gestión de } \\
\text { recursos y de estructuras de } \\
\text { apoyo }\end{array}$ & $\begin{array}{l}\text { Adecuada administración de recursos y estructuras de } \\
\text { apoyo }\end{array}$ \\
\hline
\end{tabular}

Fuente: Elaborado por autores. 